\title{
Co-ordinating Distributed Knowledge: a Study into the Use of an Organisational Memory
}

\author{
M. J. Perry ${ }^{1}$, R. Fruchter ${ }^{2}$ and D. Rosenberg ${ }^{1}$ \\ ${ }^{1}$ Department of Information Systems and Computing, Brunel University, Middlesex, \\ UK; 2Project Based Learning Laboratory, Center for Integrated Facilities Engineering, \\ Stanford University, California, USA
}

\begin{abstract}
This paper presents an ethnographically informed investigation into the use of an organisational memory, focusing in particular on how information was used in the performance of work. We argue that understanding how people make use of distributed knowledge is crucial to the design of an organisational memory. However, we take the perspective that an 'organisational memory' is not technology dependant, but is an emergent property of group interaction. In this sense, the technology does not form the organisational memory, but provides a novel means of augmenting the coordination of collaborative action. The study examines the generation, development and maintenance of knowledge repositories and archives. The knowledge and information captured in the organisational memory enabled the team members to establish a common understanding of the design and to gain an appreciation of the issues and concerns of the other disciplines. The study demonstrates why technology should not be thought of in isolation from its contexts of use, but also how designers can make use of the creative flexibility that people employ in their everyday activities. The findings of the study are therefore of direct relevance to both the design of knowledge archives and to the management of this information within organisations.
\end{abstract}

Keywords: organisational memory; engineering design; ethnography; teamwork; user appropriation

\section{INTRODUCTION}

Organisational memory is the term used for the mechanism that allows knowledge to be distributed over different individuals, time and space, providing them with the tools to access information relevant to their needs without engaging in complex search activities. Conklin [1] suggests that organisational memory, as it stands currently, is embodied in a set of documents and artefacts' (p. 561), and that this excludes collective memory - the pooled memory of individuals. However, limiting our definition of organisational memory to a single form of representational medium (documents) is not appropriate when looking into organisational information use - communication is also a vital aspect of information use [2]. Human knowledge workers carry large amounts of information about work in their heads that they use individually, collaboratively and in concert with information stored in documents.

There is support for the perspective that organisational memory is not clearly defined in Walsh and Ungson [3]. Whilst the term 'organisational memory' may not be an entity with a well defined boundary, we believe that it serves an important function in alerting us to the importance of stored information in work systems. Thus, instead of beginning the study by developing the theoretical implications of 'organisational memory', we began with the perspective that a study of its use is a more appropriate means of understanding how to support action. The paper therefore focuses on how people use information archives and distribute their knowledge across social and physical space.

Previous work into this area has focused on how people use particular technologies in developing organisational memories (e.g. [4,5,6,7]). In contrast, this study investigates how a project team manage their distributed knowledge and stored information with a broad range of non-proprietary tools. It examines how they generate, navigate and maintain information arising from their individual activities as well as through collaboration with one another, and draw from the methods of social science to do this. The study concentrates, in particular, on the use of web based tools, with a focus on advanced, yet commercially available, communications technology. 
That there is a long history to the investigation of organisational memory is evident in the literature on organisational theory [3], although its direct relationship to technology is more recent. What they do not do is to demonstrate how this feature is used. This is reflected in much of the literature, which concentrates on designing technologies for people to create and use as memories, rather than seeing what information organisations need to recall, and the mechanisms that they use to do this. We intend to show how this is done in a small organisational unit through the use of user-configured tools, which include both non-technical and technical artefacts.

Through detailed field studies, we examined what it meant for a group of co-workers to generate an 'organisational memory'. In order to look more closely at what this involved, we investigated an instance of cross-disciplinary communication in a project team, and between several different stakeholder groups. These included an engineer, architect, and construction manager, and to a lesser extent, consultants and client. When working together, they had to maintain a common understanding of the problem to discuss issues affecting their own concerns. In the team examined, the participants managed this on their own volition, by providing a record of their own activities in an electronic archive, whilst at the same time using communications technology to determine and negotiate areas of common interest.

The team's use of the technology resulted in the emergence of two important features:

a) a common informational space that they used as a resource to co-ordinate their activities, and

b) the development of new work practices and procedures to organise themselves.

The next section briefly outlines information use in organisations, examining its processing, representation in media, and mechanisms of co-ordination.

\section{INFORMATION USE IN ORGANISATIONS}

The paper presents a study of collaborative information use that is mediated through technology. This technology provides organisations - ranging in size from teams, divisions, companies and consortia with a mechanism for both managing ongoing information use, and as a means of archiving it in a manner that allows it to be recalled when required. The term used for these activities - organisational memory - is significant, in that it infers that there is a cognitive structure involved, although this exists at a multi-participant level. Conklin [1] claims that organisations have only a weak ability to learn from the past, and suggests that computers can provide a "nervous" system to augment this, to support the capture, recall and learning of knowledge. Whether or not 'memory' is used as a metaphor for understanding the processes involved, or it involves a functional cognitive architecture for collaborative problem solving, the term has entered the vocabulary of groupware design. However, we believe that the nature of the organisational memory is poorly understood, and that this has direct consequences for the design of organisational technologies.

Walsh and Ungson [3] describe many of the theoretical aspects about what it means for an organisation to have a 'memory' and whether it performs information processing in a similar way to that of an individual (its 'construct validity'). Whilst there are a number of issues regarding this construct validity, individuals in organisations require a means of accessing archived (in whatever form) information for use in their current activities. However, this perspective on the form and content of an organisational memory is only of benefit to designers if it gives them a new and useful insight into what they are attempting to support. Rather than theorise about the cognitive basis of an organisational memory, we propose that empirical investigation of such systems is more likely to be of benefit to designers.

Memory is nothing if it cannot be accessed for recall. This changes the focus of the idea of memory as a static entity into something that is a dynamic component of information processing activity. Indeed, studies from the field of distributed cognition would suggest that the structure of the representational media is of direct relevance to its processing (e.g. [8]). In such a 'cognitive' system, considering information to be seen as a memory is a potentially limiting metaphor if designers apply it without regard to its use in information processing activity. The importance of the word 'use' is emphasised here, with the implication that the context of action in organisational memory use is vital if we are to understand how to design systems that are more suited to user requirements. Studies of artefact use in context leads research away from a focused and narrow orientation on the structure and content of a 
well categorised entity to a more fluid identity. This may be less easy to examine, but has the potential to offer designers a more valuable insight into the systems' use.

An important arena for using organisational memory is in the area of engineering design - a domain involving complex knowledge structures and large amounts of information. Design is an ill-structured activity [9] with a huge number of design options, and its practitioners must navigate and structure a complex problem space. A consequence of the large size of the problem space means that it cannot be easily traversed by a single individual, but more usually involves the co-operation of a number of individuals who specialise in particular areas.

\section{FIELD STUDY OF KNOWLEDGE IN USE}

\subsection{The ethnographic approach}

The primary aim of this study is to describe and analyse the setting or context of the design and how this interplays with the goal or task that the team have to resolve. This compliments previous qualitative studies into the work of collaborative design $[10,11,12,13,14,15]$, but with a focus inclined towards the role of technology in the process, and especially its appropriation and use.

The method chosen for this study - the ethnographic approach - has a long history within social science. It is well documented elsewhere [16,17], and is only covered briefly here. Ethnography differs from experimental and laboratory based approaches that are more commonly applied in human factors research. The reason for its choice is that we are looking at an aspect of behaviour that the experimental approach is not appropriate for. In a real world environment, such as a workplace, people draw information from a huge number of sources that cannot be replicated in a laboratory setting. In particular, observations emanating from experimental studies ignore the situated and organisational features of work: people use a huge range of resources in their environments that are purposefully removed from the experimental conditions so that they do not interfere with the 'controlled variables' $[18,19]$. However, it is these excised resources, and the interactions between them, that account for much of our behaviour in the real world [20].

Ethnography was developed originally as a means of examining the lives and customs of other cultures. More recently, it has been applied by the computer science and information systems community to look at social interaction around technology [21,22,23]. Ethnography is characterised by data that is gathered from a range of sources, and emphasis is placed on its study within a context. Unlike the experimental method, the ethnographic approach allows the analyst to reveal complexity, rather than stripping it away. Van Maanen [16] describes it as being used to 'uncover and explicate the ways in which people in particular work settings come to understand, account for, take action and otherwise manage their day-to-day situation' (p. 540). In nature, it entails bringing something from the setting to the analysis, and the fieldworker attempts to understand the way that activity is understood and practised by its participants.

The intention of the ethnographic analysis is to show how work is organised [22] by the team. It has been used to examine the social organisation of groups and has become a valuable and increasingly commonly used tool in the analysis of workplace activity for systems design [21,22,24]. The detailed investigative work of the ethnographic approach to data collection allows us to examine how groups integrate and regulate both the formal and informal aspects of their work.

In this study, data was collected by the first and second authors who worked closely with the informants, observing them, doing ad hoc interviews and video-recording their meetings where possible. Over 20 hours of video material was collected, transcribed, coded and segmented. We also had access to all of the shared electronic resources used. Segments from the video material and electronic media will be presented verbatim. We believe that this perspective presents a persuasive viewpoint on the situations observed.

\subsection{The team}

This work was conducted as an investigation on the use of multimedia communications technologies in collaborative design. Access to this area was made possible through a course run at Stanford University, the 'Computer Integrated Architecture, Engineering, Construction' (A/E/C) course in the Department of Civil and Environmental Engineering. This is a learning environment that brings together different disciplines involved in construction through the use of network and information technologies $[25,26]$. The course takes a multi-site, cross-disciplinary, project-based, and team- 
oriented approach to teaching and learning [27], and engages students, faculty members, researchers, and practitioners from several disciplines. In 1997/98 the course was offered in a nation-wide pilot, where the participants were geographically distributed across Stanford University, UC Berkeley, Cal Poly San Louis Obispo, and Georgia Tech.

The field study involved a team of $\mathrm{A} / \mathrm{E} / \mathrm{C}$ students collaborating together on the design of a construction project. They included an architect, located at Georgia Tech., and an engineer, a construction manager, and an apprentice located at Stanford University. With the exception of the apprentice, all were postgraduates, with between two and ten years of industrial experience. Their task was to design a building which was owned by a 'client' (a staff member), and they were able to draw on the experience of consultants (industry based mentors with specialist skills). The team had access to a range of technologies to collaborate around, including systems such as email, web editors and workspaces, desktop video-conferencing and 3D modelling software.

The team examined was relatively small as an organisation, involving a 4 member interdisciplinary team, a client and consultants. Whilst few in number, the team formed a collaborating work unit, with many of its needs and requirements similar to those of larger organisations. Consequentially, a unit of this size provides a more focused perspective on information use because interaction between the participants can be more easily examined and their work activities followed than for a larger organisation. We recognise that larger organisations operate with more people and may exhibit different behaviours. However, we believe that whilst these behaviours are important, if an organisational memory cannot support a small organisation, then it may not be possible to apply to a larger one.

To all intents and purposes, the design project was as near to a real design task as possible. The design team had to work within tight deadlines and constraints to complete the project on budget and on time, to a high standard of workmanship. We do not however, claim that the setting is necessarily a 'typical' one. The reality is likely to be that there is no such situation, and that design projects take many forms, each unique to themselves. Nevertheless, the group studied was performing the sorts of activities that characterise design: they had to collaborate to perform problem solving, and most of the constraints that they faced were common across the industry. The non-commercial setting meant that it was possible to easily access project related information even in what might have been commercially sensitive areas.

As is common in today's knowledge intensive workplaces, the team members were engaged in multiple tasks, the $\mathrm{A} / \mathrm{E} / \mathrm{C}$ project being only one component of their ongoing work. The members had to manage their own time as individuals, and co-ordinate their schedules with the others to arrange meetings. All had offices in separate locations, although the three team members at Stanford had access to a computer room resourced with high end PC's, workstations and networking equipment. This was also where the team meetings were observed and video-recorded.

The field study took place over nine weeks, as the team was performing the second phase of the design - the detail design phase - fleshing out previous work into more detailed plans. They had already been using the technology for several weeks, and were able to configure and operate it with relative ease.

\subsection{Technologies Available}

Whilst we do not wish to concentrate on the technology alone, but on its use, it is necessary to briefly outline and describe the resources available to the team. These all came together, providing the team with a 'common information space' [28] that they could inhabit, populate and communicate through. The team members all had basic experience of these technologies and they were encouraged to configure and use them as they felt appropriate, adopting a structure and determining its content as they needed. The communications media available broadly fell into the areas of text based (email, 'chat', web pages), audio (telephone), graphical (web based images, CAD files), multi-modal (desktop videonetworking technology). The technologies themselves are considered below:

Web technology was provided in the form of a shared web workspace that all of the team could write to. They could store a variety of electronic files in this, and could access it through conventional web browsing tools. As well as text entry, images could be scanned in from paper and added to web documents, allowing sketches and photographs to be viewed on-line. 
Email allowed the team members to perform standard person-to-person, asynchronous, text based interaction. A system called 'Hypermail' allowed emails sent to a group address to be archived and viewed from the web space. These could be sorted by date, topic or author.

Audio access was provided through a telephone link. This allowed the team to make person-to-person calls, and it required, conference calls, because the device had a speaker and an external microphone. Video-conferencing software allowed audio communication, but proved to be of too low quality to be usable.

Graphical information was accessible over the web, and could be used to display slide presentations, CAD files, and scanned in photographs, jottings and sketches. These were not possible to update directly on-line, and were primarily intended for use as reference material.

The multi-modal component of the team's communications suite was provided by video-conferencing technology running over a dedicated T1 internet connection. The technology - NetMeeting - also allowed the participants to 'share' windows and applications between the two locations so that the same visual information could be synchronously seen and interacted with at two or more locations. The software also featured a 'chat' facility, so that the team could type messages to one another synchronously.

\subsection{Use of the information space}

The information space described in this section comprises of information existing over the whole design system. It includes information held by individuals as well as in the various technologies used in the design process. Whilst the technology described in the above section provided a potential for distributed information use across a network, it was not possible to know what particular aspects and configurations the team members would find useful in performing their tasks. We therefore needed to investigate how the technology was used in practice to see how the team developed, co-ordinated, and managed their distributed understandings of the developing design.

Data from the field studies are presented in five vignettes below that characterise aspects of technology use by the team. These are discussed later according to their 'dimensions of interaction': synchronicity, presence and task-based criteria.

Vignette 1: Recording discussions in chat. Meetings were the primary means used by the team to collaborate and to co-ordinate their activities. These generally took place twice a week, on Monday and Friday, lasting between 1 to 2 hours. Towards the end of the project, they became more frequent and longer, as the quantity of design information grew larger, and each discipline had to see how their own design component integrated with that of the others.

The chat window was sometimes used as a means of recording discussions in these meetings so that it could be re-used later. An example from a recorded chat session demonstrates one of the ways that they used the system:

E.g. 1 - Use of 'chat' as a permanent record of design discussions

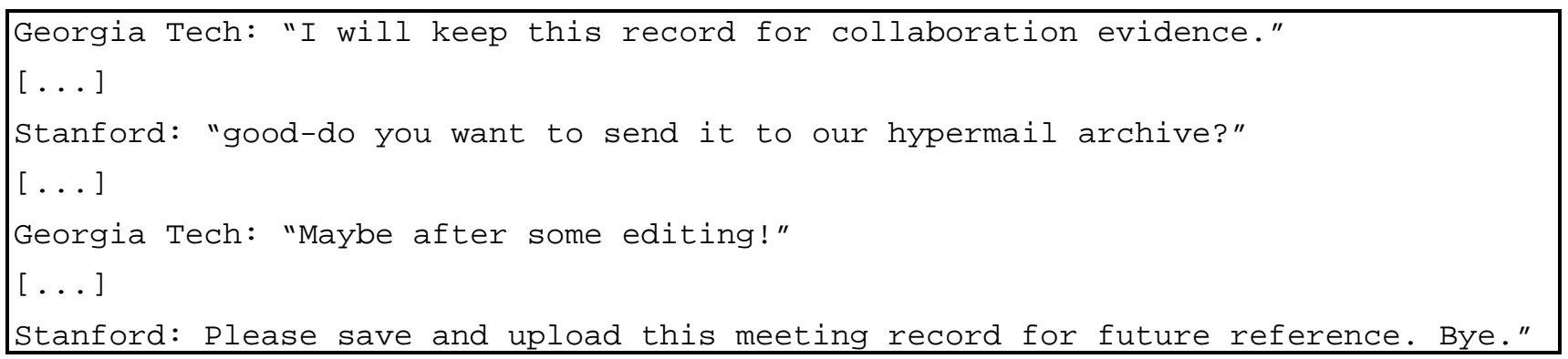

This process appears similar to the minutes that are used preserve a permanent record of a meeting's content. However, in this case, the team members did not simply use another (text based) media in parallel to the verbal channel, but took this a step further, switching their communications to a text based media for the sake of preserving its content. Interestingly, the conversation demonstrates that the archive was not necessarily a reliable record of actual events, and may be amended later. 'Chat' was also used as a supplementary channel when the telephone was not an appropriate medium, such as when conveying acronyms or technical terms. 
Vignette 2: Co-ordinating perspectives with email. Together with meetings, the other main form of communication used was email. Over the course of the project as a whole (4 months), a total of 348 email messages (between the team, as well as mentors and client) were received. The main purpose of email appears to have been that it allowed asynchronous contact that could be both sent and browsed when the sender or recipient had the time to deal with it. This, for example, could be used to deliver feedback as demonstrated in a chat meeting:

E.g. 2 - Negotiated co-ordination mechanism for providing feedback

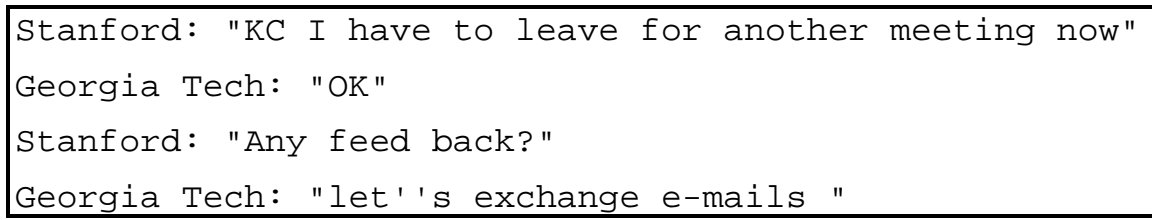

In this case email was used to communicate non-urgent messages, allowing the sender to manage their time resources more flexibly. In another example, email was also used as a means of reminding the others to perform tasks at the appropriate time - as illustrated in a quote from a chat meeting:

E.g. 3 - Mechanism for providing information awareness

Stanford: "ok, as a job comes up, you can email me the task to do"

This function of email as a "demon" was used by the team whenever they updated information in their group space that they felt the others would need to know about. These were often very brief, on many occasions having only a subject line:

E.g. 4 - Entire content of an email informing the team of an update to forum 6.

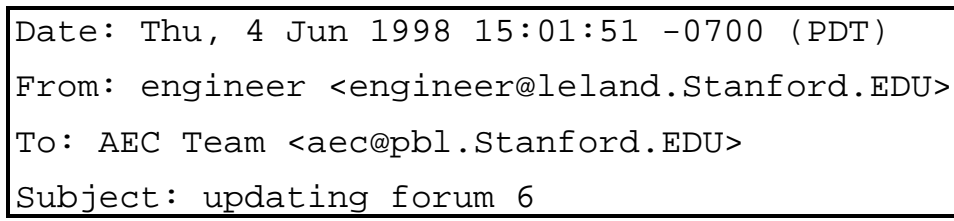

Vignette 3: Structuring the design archive. The largest and most organised repository of information that the team used lay in the 'design forums'. The team had created these themselves using the web as a platform. On the instigation of the architect, the team had built an evolving series of forums in which they could view and discuss their opinions about the design and the design process. At the time of the study, these had developed from three initial forums to four. Over the course of the field study, these grew to six. An additional, semi-permanent forum was also created for 'catch all' unstructured information. The first forum provided an area in which the team agreed protocols for discussion in the other forums, the second forum discussed the design intent, the third discussed technical issues, and the fourth discussed design alternatives. Of the final two forums, one was used to discuss the design methodology and collaboration process, the other for the final design solution.

Whilst the design forums were generated collaboratively, they were largely maintained and structured by the apprentice, who took responsibility for managing this information. To ease reading and interpretation, the team agreed to use different colours to differentiate between recent and old comments (text from forum 1):

.... I think each new person should highlight their newest comments with a color or some other feature and change any old comments to normal text, so new comments can be easily differentiated from old ones.

To demonstrate how the forums evolved, this use of colour changed, and use of a particular colour came to mean that these comments were made by a particular person, rather than as previously, to show comment recency. In addition, whenever changes were made by one of the team members to the text in a design forum, the team agreed to send out an email saying that they had done so (again, from forum 1). 
and during this time, no one else should mess with the file. This will avoid| accidental over-writing.

This demonstrates how the team developed a co-ordination protocol to avoid file conflicts (called here "over-writing"). This is very similar to Rogers' [24] description of the mediating artefacts used to avoid file conflicts in the drafting process: whenever file changes were to be made, this information was posted onto a board so that file users were aware of this. However, unlike in the Rogers study, such file conflicts were avoided, largely accidentally, because the forums were not updated frequently, occurring haphazardly around once every two or three weeks, and the chance of co-incidental simultaneous access was slight.

The design forums were intended to implement a "formalised process for documenting the design intent, concepts, perspectives, and solutions" (from the team's documentation). When questioned, the team argued that they needed them in the web format because this allowed two things. Firstly, it allowed them to undertake a structured discussion, so that the individual points could be discussed sequentially without losing focus, as might occur in a less formalised medium. Secondly, they allowed asynchronous discussions to take place, because they recognised that detailed discussions could be long and drawn out over time. By turning the discussion asynchronous, the team could spend more time on considering the details than they could in a meeting and also to reference additional information.

Vignette 4: Developing social and organisation protocols for interaction. Much of the fabric holding the group together was social and not structured in a pre-determined, or organised framework. The team had tried to specify protocols for communication, but these mechanisms were limited to basic instructions about the use of various media in specific circumstances. For example, the team members co-ordinated their (limited) shared time by agreeing to place their time schedules on the web, showing the best times for holding meetings that they could all attend. Another example drawn from the teams web space shows a protocol on how to use email so that it could be accessed over the Hypermail system; this required email to be sent from and to a particular address for it to be catalogued and archived.

E.g. 5. Instructions on the use of email.

- Use Hypermail for intra-team communication so that your communication is
archived and "project memory" is initiated.
- E-mail messages sent to mentors should be sent from pbl accounts, not leland
accounts, so that they are archived

In contrast, the team's day-to-day interactions were managed in an ongoing basis, 'on the fly', rather than on a predetermined basis. For the most part, the co-ordination procedures that the team followed were not formally defined, but were agreed upon on as needed basis. For example, there were no distinction between 'design meetings' and 'co-ordination meetings', as has been observed in other studies of design teams [15]. This might have been due to the different sizes of the teams involved, cultural differences between the USA and UK, or that the project was based in an educational setting. However, one possible reason for this was that the technology allowed co-ordination issues to be managed on an as-need basis. This relaxation in the formality of the design process was possible because the various technologies available made it relatively easy for the team members to monitor the others' ongoing work, and because a large proportion of the current design related information was visible to immediate inspection. As a consequence, co-ordination issues could be dealt with informally in regular meetings without the need for involving formal co-ordination procedures to ensure the integrity of the design solutions.

Vignette 5: Appropriation of technology for co-ordination. The team was versatile in their use of tools, using a range of devices to maintain contact with each other. Their choice of tool appeared to be as much to do with the limitations of the network, or particular circumstances, than because it was the most appropriate, for example, making use of the 'chat' system in situations where they were unable to make audio contact. In another example, a mentor (TN) attempted to co-opt an unusual technology as a communications device: 
E.g. 6. A new media for communication is adopted (from field notes)

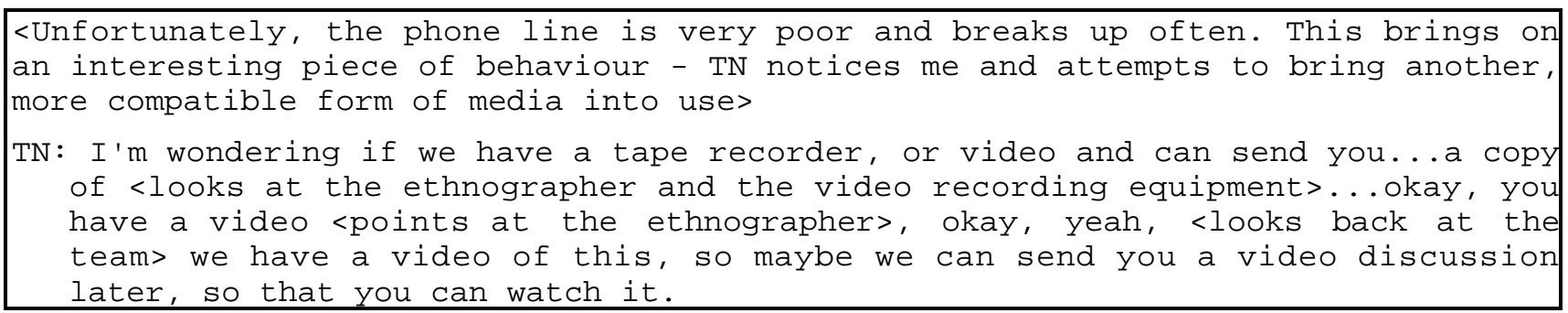

This final example demonstrates the problem of integrating all of the design records into a unified organisational memory - the range of artefacts that can be adopted and used is almost unlimited.

\subsection{Three dimensions of interaction}

Synchronicity. The technologies used by the team appear to fall into the well used categories of synchronous and asynchronous communications media, each of which is expected to fulfil a different role in collaboration. The synchronous media include the telephone, 'chat', the desktop videoconferencing link and shared window technologies, whilst the asynchronous technologies include email, the web and design forums. However, the field data shows that these distinctions become unclear when the technologies are used in real world settings and in combination with other media. For example, whilst communication using the 'chat' window is synchronous, it's value to the participants is not simply that it is synchronous, but that it also provides the team with a permanent record of the discussion. This is something that is not possible with speech, which is ephemeral. In the same way, graphics were placed on the web for asynchronous browsing, but these were commonly used as an additional resource in synchronous communication by sharing the browser window in NetMeeting. The crucial importance of this finding is that the technology configurations can change the intended status of a communications channel by putting communication into a specific context with particular resources at hand.

Presence. The communications channels and technologies can be categorised according to their spatial dimension; they can be seen as supporting either co-located or remote collaboration. Here, shared paper documents, speech (including volume and tonality), gestures, body orientation, and gaze direction contributed to communication in co-located space, whilst where the participants were distributed, technologies such as the telephone, application sharing, and video-conferencing supported distributed communication. Some of the technologies that supported distributed working carried the cues of co-located communication over space, but these were rarely able to convey the full richness of face-to-face contact.

In the study, a variety of technologies were co-opted in meetings to facilitate team co-ordination. The technology was mainly used to support distributed communication and only very rarely for co-located interaction. The significance of this is that the recorded traces left of meetings on the computer archive were biased towards discussions relating to the architect or architectural factors, the architect being the remote communicator. Even in this case, many of these meetings went unrecorded: most of the interaction took place over the telephone, and as a consequence, did not result in the creation of a document or physical archive.

The study shows that distributed teams need multi-modal communication channels to provide a context for the interpretation of remote information. Where appropriate channels do not exist, they may appropriate technologies into new configurations, such as in the use of the chat window as an archive, or more forcefully, in vignette 5, with the appropriation of the ethnographer's videotape to support their co-ordination. There appears to be a simple principle at work here, with users minimising their effort - they do not tend to bring more channels into operation than they need, but add channels when they require them. Where communications channels are deemed to be unnecessary and hard to maintain, they are often closed down. For example, whilst the team found the video-conferencing technology useful in developing an awareness of the context of the remote setting and helping them to co-ordinate their ongoing interactions, it was not deemed to be a significant part of the collaborative process. When it proved to be hard to use (usually technical difficulties), it was abandoned.

Task based interaction. There are some simple categorisations that can be made about the technologies used for particular tasks. The fieldwork demonstrates that the media used to represent 
design information are the recorded 'chat' and web technologies; explicit co-ordination was maintained through email and over the web pages; and negotiation and tacit co-ordination was facilitated through the video-conferencing technology and telephone links. Whilst these patterns of use were clearly evident from the fieldwork, this is an impoverished and over simplistic perspective that neglects to show how these media were used in combination with one another. In practice, they enriched each other, providing a context for the interpretation of information in other media. For example, although the design documentation in the CAD drawings was operated through web access, this provided a common object for discussions to take place over. Verbal discussions in another media could be 'grounded' by reference to features on the drawing, or grid locations on it. In another example, the initiation of formally arranged meetings, organised over email and the web pages, was facilitated through the use of the video-conferencing window, as a user could check whether the remote participant was present and able to attend. Use of each media could not therefore be seen as limited to a particular 'type' of task. The designers of an organisational memory must incorporate this flexibility of use into their systems.

\subsection{Summary of media use}

The fieldwork provides us with a detailed source of information about how the team members organised themselves and their data archives to implement a form of what could be called an organisational memory. It is noticeable that both internal knowledge and external media were used interchangeably. The archive was easily navigable (it could be searched in many formats and by asking other people), and it was extensible (information was dynamically added to it and the creator could be questioned). Several specific points are noted below:

- Ongoing 'chat' correspondence was used so that a permanent record existed of a synchronous collaboration. This medium was deliberately selected over audio so that a physical record was preserved. Email was used in the same way for asynchronous communication.

- Email was used as a means of distributing collaborative work over time so that shared work could be carried out when convenient to its recipients. Email could be tightly targeted at particular people and did not take up 'group time', which was a valuable commodity.

- The web was used as a means of sharing graphical representations (drawings, sketches and schedules) for referring to in conversations continued in other media. These graphical representations were usually embedded within larger text documents and were rarely revised without a detailed discussion.

- Audio technology (video-conference and telephone) was used as a transitory medium for discussing the designs over space. These could not be used as a permanent record of a meeting, and important decisions taken in these media were always followed up or confirmed with an email.

- The team members deliberately used the archive as a record of past communication. By placing their communications in a publicly accessible arena (Hypermail and the web) about what they had agreed to do, they could see what responsibilities they had taken on, and what they believed other people should be responsible for.

- Communication protocols were hard to establish in an on-line presence, and the group tried to formalise these in an organised manner by publishing a set of 'rules' that explained how the system was to be used for asynchronous communications. In synchronous communications, these were negotiated as required.

- Cross-referencing across media was common, as different media were used in conjunction with one another as the benefits and limitations of them became apparent to the participants. This was context dependent and was supported by the range of technologies that allowed flexibility of use.

This summary of the activities performed by the team can only capture a very small part of their involvement with one another. However, it shows how the team developed a means of collaborating that allowed its members to draw upon each other's knowledge as well as from existing artefacts.

\section{KNOWLEDGE, MEMORY AND TECHNOLOGY IN USE}

The focus of the fieldwork is on specific instances of the use and communication of knowledge by the team, the storage and retrieval of generated design material, and the use of technology to support these activities. This perspective gives an insight into the more general aspects of information use and the 
importance of technology in this, so that issues relating to 'organisational memory' can be more clearly identified.

The critical finding from the fieldwork is that of structure. Structure - the protocols and practices applied to the work - was integral to the way that the knowledge archive and communications networks were used and integrated with one another. The different structures that the team applied were derived from constraints in the technology, from the time available, from the geographical distance between participants, as well as constraints negotiated as organisational procedures and determined by social pressures. However, it is not the reasons for these structures that we are interested with per se, but how they were used by the team members to organise their collaborative activities and to achieve their shared goals.

Traditionally, the engineering design process has involved the development and use of structured, paper based archives which include drawings, diaries, project log-files and 'request for information' systems. These are highly controlled means of storing and ordering information that are regularised and monitored, in terms of content and who they are read by. However, in the system observed, this was not the case. The structuring process of the electronic media was largely ad hoc, and not organisationally formalised. In many cases, this was not a problem for the users because the archive was not large enough to cause problems in locating information. However, problems were observed when information had to be located and displayed quickly: time was wasted as the communicants had to 'surf' the electronic archive to look for particular information.

Structuring the organisation of the electronic design archive was not a trivial activity. The structure of the archive was not static, determined at the beginning of the project, but ongoing and dynamic. New aspects of the design did not always 'fit' into parts of the old structure in such a way that they could be easily located. This occurred because of the 'ill-structured' nature of design [9], in which not enough is known about a problem from its initial conditions to be able to determine a completely specified set of future outcomes. This situation is common in other forms of work where the decision path for a particular project is contingent on underspecified starting conditions.

The fieldwork provides several examples that illustrate the 'user-structuring' that had to be performed to enable information location. As the design developed over time, new information was continually added to the design archive, all of which was accessible over the computer network from the group space. However, the addition of this new information entailed restructuring the design archive by the team, changing the categories with which they organised information. Retaining a single archive structure throughout the design process over time was not always possible, and after the addition of new information, the organisation of the site was usually changed in some way. This occurred as the team wished to retain old, out of date information that was important for reference - they had to create a new 'old links' section on their web page that they could use for accessing it. Another example of dynamic user structuring occurred in the two different uses of colour in the forums, first to highlight time information, and then later, to denote authorship: the structure of the site was changed over time, according to the users' circumstances and needs.

It may not be possible for the archive creators to manage to categorise new design information in a simple or rational way within the original structure. The developers of the archive are therefore left with the problem of either having to:

a. change the interface to incorporate the new information in a meaningful way (thus a problem for navigation in reducing the archive's structural consistency), or

b. categorising this information in an unnatural way to force it into the existing archive structure (a problem for navigation by decreasing contextual cues about its content).

When several people are involved in this process (in a collaboratively maintained archive), the inconsistent approaches selected can make navigation even more problematic. Within the team studied, this particular problem was resolved by a single person (the apprentice) being designated to maintain the webspace.

Whilst the structure of the electronic archive was an important aspect of the design work, the design information represented in the electronic group space was not the design - that is, the archive was not a record of the actual design process. Rather, it was a post hoc rationalisation of the process, constructed by the team, and which was even occasionally appropriated by the team for a different end. In addition to its use in design, the archive was also used to demonstrate the 'professionalism' of the team 
members to their tutor. They therefore did not always include information that showed their uncertainty about their design solutions in the archive. This was intended to make themselves appear more confident about their solutions; for example, emails considered to be 'uncertain' were not always forwarded to the Hypermail archive (see example 1). In other cases, parts of the design were either considered too 'simple' to be included in the archive, or were implicitly agreed upon. In some instances, decisions were made in media that did not transfer easily into the archival media - from face to face meetings, CAD and the 3D modelling software, and these were only briefly summarised in the archive. For this reason, although the archive appeared to be a complete record of the design, it was an abridged and expurgated version, created and used for reasons additional to the technical process of design. Of course, in the real world, design is not a purely technical activity, and is embedded in a social fabric that encapsulates political, motivational and other aspects (e.g. [29]).

\section{IMPLICATIONS FOR TECHNOLOGY}

The fieldwork has much to say about the development and use of what can be called organisational memory. These findings suggest a number of areas that relate to the development of organisational technologies to support collaborative information use. One of the problems noted by the participants in the study was that they found it difficult to co-ordinate their often competing objectives. Whilst architects, engineers and construction managers are all involved in the design process, their areas of expertise and responsibility differ. Each needed to communicate the results of their work to the others and co-ordinate their efforts to produce a final product. Although training and experience are one means of reducing confusion in this area, the prospect of technology that can help to manage this is a highly desirable one. Examining how people structure their working practices can provide an insight into where these difficulties lie, and identify strategies for their resolution. By drawing from existing user practices, we have a basis for developing methods that are minimally intrusive into the work itself.

The problem of co-ordinating different user objectives was exacerbated by having a large and expanding physical design archive. Use of the computer was seen as the key to this, because of the powerful storage, search and communicative value that they brought to the management of the archive. However, by themselves, the existing computer tools did not offer the flexibility and customisation that the team members required for their ongoing work. In order to manage the increasingly complex system of documents and other information, the team developed working practices and organisational procedures for document management that enabled them to make use of this archive in a way that suited their requirements. This highlights a danger in developing technology to support 'generic' coordination practices. These may increase the rigidity of the system, by hindering the powerful social mechanisms groups develop in response to specific co-ordination problems. This is not to suggest that designers should ignore the role of co-ordination in developing technology, but that they should not exclude users from being able to use social mechanisms to augment the processes and constraints within the technology.

In addition to maintaining a repository of current design information, the team also needed to keep a record of 'old' design information, for example to re-use it, or to try to understand why the design had developed in a particular direction. A problem arose in this - the structure of the archive changed as its content changed. Where structural change to an archive occurs, information cannot be found in its original location, and may be as hard to locate as to recreate from scratch. This needs to be considered when updating the archive; the team studied found it to be a particularly difficult problem to resolve, and never fully achieved a solution to it. Larger teams, working over longer time frames, and producing more documentation are likely to find this even harder to solve. An alternative method of changing or formalising aspects of the archival process may provide a solution to this structure-content dynamic. Planning the structure of the archive so that the categorisation structures are extensible would seem an important element to consider in its early conception. The technology developed should not hinder this extensibility by enforcing too rigid an informational structure on its users.

The development of communications technology has made it increasingly possible for collaborating teams to work in non-contiguous locations. However, whilst the potential exists for distributed collaboration, in practice, performance is likely to suffer if there is no corresponding improvement in support for co-ordinating the perspectives of the participants. These problems are not insurmountable, and the team studied developed social co-ordination mechanisms around the constraints imposed by the technology. This study shows how existing technologies are appropriated by users in novel ways to support their ongoing interactions. We have observed how co-ordination is managed on a largely $a d$ 
hoc basis, and the flexibility of the technologies to be co-opted for different activities appears to be critical in this. Whilst this has proved to be successful for a small team, bringing together larger groups, or several teams who may use multiple techniques for managing their co-ordination may result in confusion, and a less satisfactory outcome.

\section{DISCUSSION}

The media used by the team were of two very distinct kinds - media for communicating with, and media for providing a background to communication. Most obviously, the email and videoconferencing technology was used for communicating with people, whilst material on the web pages formed an archive that provided a context around which the communications could take place. This archive could be called up, pointed to, referred to in communication, or browsed independently. Whilst these two forms of technology for direct communication and archival were distinct, they should not be considered independently: each provided the other with information about how they were to be interpreted. In some cases, this link was explicit and direct - the Hypermail records of email and the 'chat' logs were both a means of communication and its own archive.

Conklin [1] claims that a problem that most organisations face is not that they fail to collect information and artefacts, but that they lose the context (or 'rationale') that lies behind their generation. The designers of complex systems are drowning in a sea of unstructured and semi-relevant information. He concludes that organisations need to move towards a new paradigm of information management, focused on the 'process' of information generation and use. Whilst Conklin [4] uses this argument as a means of developing a particular technology (gIBIS), the participants in this study have achieved this themselves, appropriating the various forms of communications technology to demonstrate the processes behind their information generation. There were a number of possible reasons why this has happened. Perhaps more important than discussing how or why they did this is the simple observation that they managed to do so without recourse to a technology developed specifically for managing an organisational memory, producing instead an emergent structure fashioned from social as well as technological components.

By appropriating technologies for particular functions, the team studied developed a highly effective archive of previous interactions - their 'organisational memory'. Their success is due to the adoption of a 'rhetorical method' [1] that gave consistency to the diverse content of their design archive". This is a method of structuring interaction and may exist either as a constraint within the technology or develop socially through interaction. We see both of these features expressed in the fieldwork. As designers, we can manipulate the technical constraints to support particular activities, and we can also provide guidelines for practice (such as in the use of colour in vignette 3). Communications technologies are now relatively mature, and it is the role that the organising structures play in organisational memory that increasingly must become the focus of research. Users will appropriate technology to their own agendas, and to expect the technology to be used in a standard way fails to account for the subsidiary role that it plays in work performance - work should not be built around the technology, but should allow users to adapt it to the needs of the task.

This research is intended to show how individuals and group structure their activities to create a productive work environment. The system developed by the participants incorporated stand alone technologies, configurations (or 'suites') of technologies, and managed systems of use for integrating these technologies into their work processes. Indeed, the organisation of the team around their work, and the use of technology to support group interaction appear to be deeply entwined with one another. If we are to consider the team's use of an organisational memory, the technology and the organisational context that it is used within cannot sensibly be seen as distinct. Technology by itself, it seems, cannot act as a memory without an organisational system that allows it to be used as such.

\footnotetext{
* The rhetorical method is one of three components that Conklin claims are required for an organisational memory, the others being hypertext and groupware. Hypertext allows the non-linear recall of information, flexibly organised enough to support ongoing restructuring and expansion. Groupware captures the semi-structured records of computer communications so that they can be shared later.
} 


\section{ACKNOWLEDGEMENTS}

This work was partially supported by the CICC project (ACTS project no. AC 017). The study was also carried out whilst the first author was working as a visiting scholar at CSLI (the centre for the study of language and information) at Stanford University. The authors would like to thank the A/E/C team who allowed us to work with them so closely.

\section{REFERENCES}

1. Conklin, E.J. Capturing organisational memory. In Readings in groupware and computersupported cooperative work: assisting human-human collaboration. Morgan Kaufman: San Mateo, California, 1993, pp 561-565.

2. Tuomi, I. The communicative view on organizational memory: power and ambiguity in knowledge creation sytems. In Proceedings of the 29th Annual Conference on System Sciences. IEEE Press, 1996, pp 147-155.

3. Walsh, J.P. and Ungson, G.R. Organizational memory. Academy of Management Review, 1991; 16: 57-91.

4. Conklin, J. \& Begeman, M.L. gIBIS: a hypertext tool for exploratory policy discussion. In the Proceedings of the Conference on Computer-Supported Cooperative Work. ACM Press, 1988, pp 140-152.

5. Berlin, L.M., Jeffries, R., O’Day, V., Paepcke, A. \& Wharton, C. Where did you put it? Issues in the design and use of a group memory. In Proceedings of INTERCHI - Human Factors in Computer Systems. ACM Press, 1993, pp 23-30.

6. Terveen, L.G. Overview of human-computer collaboration. Knowledge-based Systems, 1995; 8 (2-3): 67-81.

7. Ackerman, M.S. Augmenting the organizational memory: a field study of Answer Garden. In the Proceedings of the Conference on Computer-Supported Cooperative Work. ACM Press, 1994, pp 243-252.

8. Hutchins, E. Cognition in the Wild. MIT Press: Bradford, 1995.

9. Simon, H.A. The structure of ill-structured problems. Artificial Intelligence, 1973; 4: 181-204.

10. Bucciarelli, L.L. Designing engineers. MIT Press: Cambridge, Mass, 1994.

11. Bucciarelli, L.L. An ethnographic perspective on engineering design. Design Studies, 1988; 9 (3): 159-168.

12. Murray, D. An ethnographic study of graphic designers. In Proceedings of the third European Conference on Computer-Supported Cooperative Work. Kluwer Academic Press: Netherlands, 1993, pp 295-309.

13. Pycock, J. \& Bowers, J. Getting others to get it right: an ethnography of design work in the fashion industry. In the Conference on Computer-Supported Cooperative Work. ACM Press, 1996, pp 219-228.

14. Perry, M. Process, representation and taskworld: distributed cognition and the organisation of information. In Proceedings of ISIC'98 - Information Seeking in Context: an International Conference on Information Needs, Seeking and Use in Different Contexts, 1999 (In press).

15. Perry, M. \& Sanderson, D. Co-ordinating joint design work: the role of communication and artefacts. Design Studies, 1998; 19 (3): 273-288.

16. van Maanen, J. The fact of fiction in organisational ethnography. Administrative Science Quarterly, 1979; 24: 539-550.

17. Agar, M.H. The professional stranger: an informal introduction to ethnography. London: Academic Press, 1980.

18. Anderson, B. Button, G. \& Sharrock, W. Supporting the design process within an organisational context. Proceedings of the third European Conference on Computer-Supported Cooperative Work. Kluwer: Netherlands, 1993, pp 47-59. 
19. Norman, D.A. Cognition in the head and in the world - an introduction to the special issue on situated action. Cognitive Science, 1993; 17: 1-6.

20. Suchman, L. Plans and Situated Actions. Cambridge: CUP, 1987.

21. Heath, C. \& Luff, P. Collaborative activity and technological design: task coordination in London Underground control rooms. In Proceedings of the 2nd European Conference on Computer Supported Cooperative Work. Kluwer Academic Publishers: Netherlands, 1991, pp 65-80.

22. Hughes, J.A., Randall, D. \& Shapiro, D. Faltering from ethnography to design. CSCW 92: sharing perspectives. Proceedings of the Conference on Computer Supported Cooperative Work. ACM Press, 1992, pp 115-122.

23. Button, G. \& Sharrock, W. The production of order and the order of production. In Proceedings of the 5th European Conference on Computer Supported Cooperative Work p.1-16. Kluwer Academic Publishers: Netherlands, 1997.

24. Rogers, Y. Coordinating computer-mediated work. Computer Supported Cooperative Work, 1993; 1: 295-315.

25. Fruchter, R. The A/E/C Virtual Atelier: Experience and Future Directions. In Proceedings of the 4th Congress of Computing in Civil Engineering, American Society of Civil Engineers: Boston. 1998, p.395-402.

26. Fruchter, R. and Reiner, K. ProMem: Project Memory for Shared Design Evolution Capture. In Proceedings of the Structural Engineers World Congress (1998), (published on CDROM), 1998.

27. Fruchter, R. Roles of computing in $\mathrm{P}^{5} \mathrm{BL}$ : problem-, project-, product-, process-, and people based learning. Artificial Intelligence for Engineering Design and Manufacturing, 1998; 12: 6567.

28. Bannon. L. \& Bødker, S. Constructing common information spaces. In Proceedings of the 5th European Conference on Computer Supported Cooperative Work. Kluwer Academic Publishers: Netherlands, 1997, pp 81-96.

29. Latour, B. Aramis - the Love of Technology. (Trans. C. Porter). Harvard University Press: Cambridge, MA. 1996. 